\title{
TÉCNICAS DE INTELIGENCIA ARTIFICIAL PARA OPTIMIZAR LA EFICIENCIA DEL PROCEDIMIENTO DE SELECCIÓN PARA LA CONTRATACIÓN DE OBRAS PÚBLICAS*
}

El presente artículo propone el diseño de un modelo que proporciona una arquitectura genérica que actúa en forma autónoma en los procedimientos de selección en la contratación de obras públicas, generando un criterio de decisión autómata en caso de empate. Para el procedimiento de selección Adjudicación Simplificada, en caso de empate, se propone la elección del postor mediante un sorteo electrónico basado en un sistema de aleatoriedad controlada de encriptación y transformación. Para el procedimiento de selección Licitación Pública, en caso de empate se propone la elección del postor mediante un índice de cumplimiento pronosticado de acuerdo con el comportamiento de las empresas en la ejecución de proyectos de infraestructura similares. Con este fin se genera un modelo que realiza la predicción de la probabilidad de éxito o fracaso del postor de ejecutar el proyecto antes de iniciarlo, usando redes neuronales artificiales como herramienta de análisis. En el presente documento se revisan las características comunes de las redes neuronales artificiales.

Palabras clave: redes neuronales artificiales, toma de decisiones, riesgo

\section{Abstract}

\section{Artificial intelligence techniques for optimizing the efficiency in public works contracting selection processes}

The present article proposes to design a model that provides a generic architecture which acts autonomously in public works contracting selection processes, in order to generate an automated decision criterion in the event of a tie. For the Simplified Tender selection process, in case of a tie, it is proposed to choose the bidder by means of an electronic lottery based on a controlled randomization system of encryption and transformation. For the Public Bidding selection process, in the event of a tie, the bidder is chosen by means of a predicted compliance index according to the behavior of the companies when executing similar infrastructure projects. To this end, a model that predicts the probability of success or failure of the bidder to execute a project before initiating it is generated, using artificial neural networks as an analysis tool. This paper reviews the common characteristics of artificial neural networks.

Keywords: artificial neural networks, decision-making, risk

* Este artículo deriva del proyecto de investigación "Modelo de gestión de las compras públicas en proyectos de infraestructura, basado en técnicas de inteligencia artificial para optimizar la eficiencia del sistema de contrataciones de obras en el Estado Peruano", del Instituto de Investigación Científica de la Universidad de Lima. 


\section{Introducción}

La visión actual de impulsar procesos innovadores en las compras públicas es un nuevo enfoque que supone alinearse con la tendencia mundial de las contrataciones públicas, dejando atrás la gestión centrada en procedimientos legalistas y burocráticos, para concentrarse en la obtención de resultados y en asignar como rol de la tecnología el de generar entornos inteligentes. Mediante las técnicas de inteligencia artificial y en un marco legal adecuado es posible contribuir con la gestión de las instituciones públicas en el cumplimiento de sus objetivos, procurando un servicio oportuno y transparente.

El presente artículo contribuye a esta visión y propone una arquitectura genérica que actúe en forma autónoma en los procedimientos de selección de contratación de obras públicas, generando un criterio de decisión autómata en caso de empate, en los procedimientos de selección Adjudicación Simplificada y Licitación Pública, aplicados para la contratación de obras públicas. Asimismo, se propone la integración de información para el procedimiento de selección mediante una base de datos centralizada para facilitar el registro y selección de tal forma que el procedimiento de selección de empresas se haga de manera automática. Para los procesos de Adjudicación Simplificada, se propone la elección del postor mediante un sorteo electrónico basado en un sistema de aleatoriedad controlada basada en el método aleatorio SHA512 de encriptación y la transformación del hash. Para el procedimiento de selección bajo la modalidad de Licitación Pública, en caso de empate, se propone la elección del postor mediante un índice de eficiencia pronosticado por un sistema de acuerdo con el comportamiento de las empresas en la ejecución de proyectos de infraestructura similares, donde se genera un modelo que realiza la predicción de la probabilidad de éxito o fracaso del postor de ejecutar el proyecto antes de iniciarlo. Esto supone el pronóstico del índice de eficiencia de cada uno de los postores que participan en el proceso de selección del proyecto, el cual servirá para realizar un desempate en caso de que algunos postores igualen en el puntaje final. También implica pronosticar el índice de eficiencia del postor ganador de la buena pro respecto a su probabilidad de éxito o fracaso en el proyecto, realizando un replanteo de estas probabilidades, comparando los datos históricos con el comportamiento del postor respecto de los riesgos identificados en el proceso de negocio. Para tal fin se usan las redes neuronales artificiales como herramienta de análisis. En el presente documento se revisan las características comunes de las redes neuronales artificiales.

\section{Fundamentos teóricos}

\subsection{Selección aleatoria y verificación con el método de encriptación SHA256}

El algoritmo propuesto es una variante del Provably Fair, implementado y explicado por bitzino.com, en forma de un sistema para poder procesar una bajada de cartas de manera transparente. Es un modelo del cual se diversifican muchas variantes que actualmente se 
usan en casinos virtuales, en especial los que usan criptomonedas como dinero (Bitcoin, LiteCoin, LottoCoin, DogeCoin, Matonis, 2012). En el white paper publicado en el techblog de Bitzino se explica el algoritmo que usan en el casino. Si bien no fue el primer uso de un algoritmo de esta naturaleza, fueron pioneros en presentar la elaboración del sistema de forma transparente. Este algoritmo Provably Fair se basa en la participación tanto del cliente como del servidor para generar una cadena encriptada única y representativa del resultado, la cual el cliente podría ver antes de confirmar su participación en el sistema. De esta forma, el cliente podría verificar el resultado obtenido y los parámetros que ingresó con la cadena que recibió. Así podría saber desde el principio que los datos no fueron manipulados y que por lo tanto fue un proceso $100 \%$ justo. Por la naturaleza de las apuestas, los casinos que optan por usar este sistema agregan una cadena especial secreta que solo es revelada luego de la confirmación del usuario en el sistema, ya que un tercero podría intentar hacer una ingeniería en reversa del proceso y evaluar la apuesta.

La variación que se propone en este documento no considera necesario el empleo de una cadena secreta. Los datos que se usarán en la evaluación de desempate de los postores solo se modifican con la participación de estos. Por eso es posible presentar toda la información de la evaluación automática en todo momento y ser $100 \%$ transparentes con los usuarios.

El método de encriptación propuesto es el SHA256, que genera cadenas de 256 bits, las cuales permiten que las colisiones entre evaluaciones sean virtualmente imposibles (la probabilidad de colisión es de 1/(2256)). La encriptación SHA256 pertenece al algoritmo de encriptación SHA 2. Es un algoritmo de encriptación para la verificación de datos y es destructivo, lo que significa que no se puede reconstruir la data a partir del hash outcome del SHA 2 (no se puede reconstruir, pero sí se puede identificar). En el algoritmo el mensaje se divide en segmentos de tamaño constante y cada segmento se somete a una serie de operaciones lógicas cuyos resultados se concatenan y forman el hash verificador. Este algoritmo de encriptación fue patentado por la NSA de Estados Unidos y tiene una declaración de licencia libre de derechos (consultar los enlaces [http://worldwide. espacenet.com/publicationDetails/biblio?CC $=$ US\&NR=6829355\&KC=\&FT=E\&locale $=$ en_ EP] y [https://datatracker.ietf.org/ipr/858/]).

$$
\begin{aligned}
& \operatorname{Ch}(X, Y, Z)=(X \wedge Y) \oplus\left(X^{\prime} \wedge \mathrm{Z}\right), \\
& \operatorname{Ma}(X, Y, Z)=(X \wedge Y) \oplus(X \wedge Z) \oplus(Y \wedge Z), \\
& \Sigma 0(X)=\operatorname{RotR}(X, 2) \oplus \operatorname{RotR}(X, 13) \oplus \operatorname{RotR}(X, 22), \\
& \Sigma 1(X)=\operatorname{RotR}(X, 6) \oplus \operatorname{RotR}(X, 11) \oplus \operatorname{RotR}(X, 25), \\
& \sigma 0(X)=\operatorname{RotR}(X, 7) \oplus \operatorname{RotR}(X, 18) \oplus \operatorname{ShR}(X, 3), \\
& \sigma 1(X)=\operatorname{RotR}(X, 17) \oplus \operatorname{RotR}(X, 19) \oplus \operatorname{ShR}(X, 10) \\
& \wedge==\operatorname{AND} \\
& \oplus==X O R \\
& V==O R
\end{aligned}
$$


RotR $==$ Desplazamiento circular de bits a la derecha

$\mathrm{ShR}==$ Desplazamiento de bits a la derecha

$'==$ Complemento

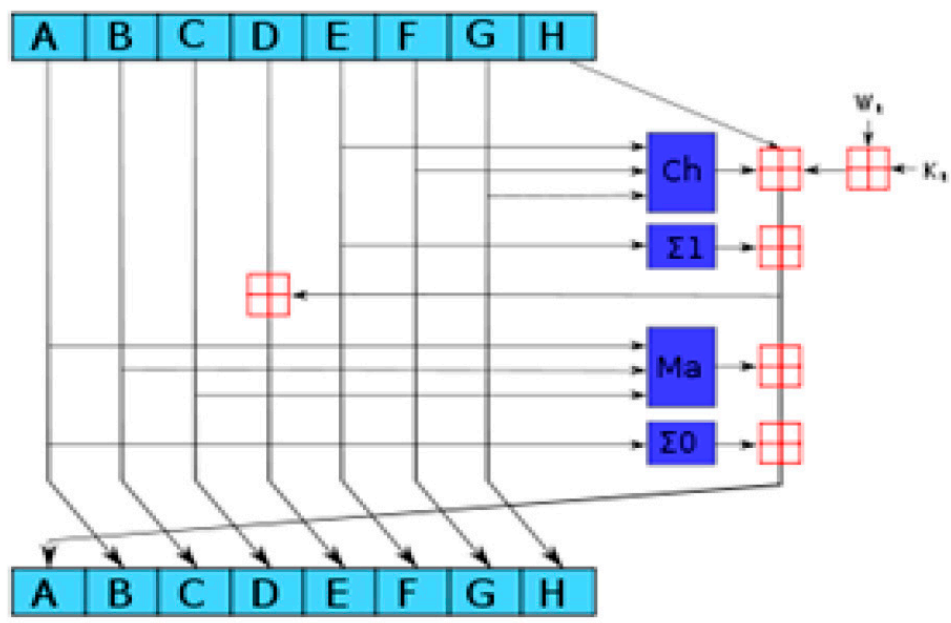

Figura 1. Algoritmo

Elaboración propia

Este se aplica en 64 segmentos de 32 bits pertenecientes a la cadena a encriptar y luego se vuelven a juntar los segmentos una vez encriptados para formar el hash representativo de esta.

Esta es una encriptación destructiva representativa por lo que no se toman absolutamente todos los segmentos de entrada, sino que se seleccionan los primeros 16 y el resto se une sumándolo con el siguiente patrón (donde Wi es el segmento i):

$$
\mathrm{Wi}=\sigma 1(\mathrm{Wi}-2)+\mathrm{Wi}-7+\sigma 0(\mathrm{Wi}-15)+\mathrm{Wi}-16,17 \leq \mathrm{i} \leq 64 .
$$

Luego de la separación en segmentos se aplica el algoritmo en 64 rondas para luego ser concatenados de vuelta y formar el hash representativo.

SHA256: (http://www-ma2.upc.es/ cripto/Q2-06-07/SHA256english.pdf) es un método de encriptación estandarizado del que existen implementaciones en muchos lenguajes de programación, por lo que no es necesario volver a programar el algoritmo. No es posible desencriptar la información sin que se pierda; sin embargo, es $100 \%$ confiable para la verificación de datos. Requiere una computación mediana, por lo que se debe aumentar el requerimiento de recursos para una implementación a gran escala. 


\subsection{Redes neuronales artificiales}

Las redes neuronales artificiales forman parte de un conjunto de metodologías que se usan como soporte de trabajo de las organizaciones inteligentes y que actualmente tienen la denominación de "análisis avanzado". Las redes neuronales artificiales no son programadas, aprenden a partir de ejemplos. Normalmente, a una red neuronal artificial le presentamos una serie de patrones ejemplos a través de los cuales ella debe aprender. Debido a que el aprendizaje es por ejemplos, las redes neuronales artificiales tienen un gran potencial para crear sistemas de computación que no necesitan ser programados. Esto supone un enfoque radicalmente distinto a los clásicos sistemas de software de desarrollo. En los programas de cómputo, cada paso que el computador ejecuta debe ser previamente especificado por el programador, considerando que cada paso del proceso absorbe tiempo y recursos. Las redes neuronales comienzan con ejemplos de entradas y salidas y aprenden a producir la salida correcta para cada entrada. El enfoque de las redes neuronales consiste en que no se requiere identificación de características ni el desarrollo de algoritmos y programas para la resolución de problemas particulares. Sin embargo presenta dos desventajas: el tiempo de aprendizaje de la red no puede ser conocido a priori, y el diseño de una red por prueba y error puede ser muy complejo. Esto quiere decir que mientras la red no haya concluido su proceso de aprendizaje no puede ser descartada (Isasi y Galván, 2004).

Actualmente existen variados modelos de redes neuronales artificiales que son usados en diversos campos de aplicación, destacando el modelo de Michie, Spiegelhalter y Taylor (1994) que es considerado el estudio comparativo más completo entre redes neuronales artificiales y modelos estadísticos. orientado a la clasificación (Sarle, 2002); el modelo de la red de propagación hacia atrás (Rumelhart, Hinton y Williams, 1986) y los mapas autoorganizados de Kohonen (1982). Estas estructuras pueden ser utilizadas en la clasificación y predicción del comportamiento de sistemas no lineales con información borrosa o incompleta, como es el caso del proceso de toma de decisiones.

\section{Definición del problema de investigación}

Uno de los problemas principales que enfrenta el Estado en las compras públicas, en lo que respecta a la contratación del proveedor para la ejecución de una obra de infraestructura pública, es tener un sistema de selección de proveedor eficiente (buena pro) que garantice la transparencia, disminuya la dilación del proceso por la interposición de recursos de apelación, permita un aumento de la cantidad de proveedores y acierte en la selección del mejor postor a fin de evitar riesgos futuros durante la ejecución de la obra.

La ley de contrataciones del Estado peruano y su reglamento (Ley 30225 y su última modificatoria aprobada por decreto legislativo 1341, Ley 29873, y la modificatoria del reglamento aprobada por decreto supremo 056-2017-EF, que entró en vigencia el 3 de abril del 2017), en sus artículos 32 
a 69, establece la estructura, disposiciones generales, bases y procedimientos de los procesos de selección. Los artículos 32, 49, 57 y 66 del Reglamento de Contrataciones del Estado expresan los procesos de selección y sus procedimientos aplicables a la ejecución de obras públicas, como son la Adjudicación Selectiva y Licitación Pública. Una referencia respecto a los montos aplicables a los procesos de selección descritos en obras públicas se observa en la figura 2.

\begin{tabular}{|c|c|c|c|c|c|}
\hline \multirow[b]{2}{*}{ PROCEDIMIENTO DE SELECCIÓN } & \multirow[b]{2}{*}{ BIENES } & \multicolumn{3}{|c|}{ SERVICIOS } & \multirow[b]{2}{*}{ OBRAS } \\
\hline & & $\begin{array}{l}\text { SERVICIOS } \\
\text { EN GENERAL }\end{array}$ & $\begin{array}{l}\text { CONSULTORÍA } \\
\text { EN GENERAL }\end{array}$ & $\begin{array}{l}\text { CONSULTORÍA } \\
\text { DE OBRAS }\end{array}$ & \\
\hline LICITACIÓN PÚBLICA & $>=S / .400000$ & & & & $>=S / .1800000$ \\
\hline CONCURSO PÚBLICO & & & $>=S / .400000$ & & \\
\hline ADJUDICACIÓN SIMPLIFICADA & $\begin{array}{l}<\mathrm{S} / .400000 \\
>\mathrm{S} / .33200\end{array}$ & & $\begin{array}{l}<S / .400000 \\
>S / .33200\end{array}$ & & \\
\hline SELECCIÓN DE CONSULTORES INDIVIDUALES & & & $\begin{array}{l}<=S / .40000 \\
>S / .33200\end{array}$ & & \\
\hline SUBASTA INVERSA ELECTRÓNICA & >S/. 33200 & >S/. 33200 & & & \\
\hline COMPARACIÓN DE PRECIOS & $\begin{array}{l}<=S / .62250 \\
>S / .33200\end{array}$ & $\begin{array}{l}<=S / .62250 \\
>S / .33200\end{array}$ & & & \\
\hline \multicolumn{6}{|c|}{$\begin{array}{l}\text { Fuente: } \\
\text { Artículo } 16 \text { de la Ley N }{ }^{\circ} 30693 \text {, Ley de Presupuesto de Sector Público para el año fiscal } 2018 . \\
\text { Artículos del } 22 \text { al } 25 \text { de la Ley N }{ }^{\circ} 30225 \text {, Ley de Contrataciones del Estado. } \\
\text { Literal a) del artículo } 5 \text { de la Ley de Contrataciones del Estado. } \\
\text { Decreto Supremo } N^{\circ} 380-2017-E F \text { que aprueba el valor de la UIT durante el año } 2018 .\end{array}$} \\
\hline
\end{tabular}

Figura 2. Montos establecidos por proceso de selección

Fuente: Organismo Supervisor de las Contrataciones OSCE. Montos para los procedimientos de Selección. Año Fiscal 2018.

Si bien el Reglamento de Contrataciones del Estado establece en sus artículos 32 a 69 una normalización para la determinación del tipo de proceso, las etapas del proceso, los requisitos de calificación y los factores de evaluación del proceso, debe tenerse en cuenta que algunos parámetros de los requisitos de calificación, como equipamiento estratégico, formación académica, experiencia del postor en obras similares y generales, están sujetos al criterio técnico del área usuaria de cada entidad estatal, el cual debe procurar no requerir características, años de antigüedad y demás condiciones que no se hayan previsto en el expediente técnico o que constituyan exigencias desproporcionadas, irrazonables o innecesarias.

Ahora bien, el artículo 54.6 del Reglamento de la Ley de Contrataciones del Estado (RLCE) establece que en el supuesto de que dos o más ofertas obtengan el mismo puntaje, obtiene el mejor orden de prelación aquella oferta más cercana por debajo del promedio. En caso de empate en puntaje y en oferta económica, el orden de prelación se determina por sorteo. El artículo 54.7 
del RLCE indica asimismo que para la aplicación del sorteo se requiere la participación de notario o juez de paz y la citación oportuna a los postores que hayan empatado.

Respecto al procedimiento de selección de adjudicación simplificada, el artículo 69 del RLCE establece que en caso de empate, tratándose de obras, en el supuesto de que dos o más ofertas empaten, la determinación del orden de prelación de las ofertas empatadas se efectúa siguiendo estrictamente el siguiente orden: a) las microempresas y pequeñas empresas integradas por personas con discapacidad o los consorcios conformados en su totalidad por estas empresas, siempre que acrediten tener tales condiciones de acuerdo con la normativa de la materia; b) las microempresas y pequeñas empresas o los consorcios conformados en su totalidad por estas, siempre que acrediten tener tal condición de acuerdo con la normativa de la materia, o c) a través de sorteo. Para la aplicación del último criterio de desempate se requiere la citación oportuna a los postores que hayan empatado.

Observamos pues que la aplicación del sorteo como medio de desempate no asegura la elección del postor más idóneo para la ejecución de la prestación y, de otro lado, la realización del sorteo no contiene un procedimiento específico que asegure su transparencia, toda vez que no expresa un procedimiento que pueda implementarse en un sistema autónomo, de manera que el proceso se realice en forma virtual y verificable sin requerir la presencia de las partes.

En los procesos de selección de envergadura, como las licitaciones públicas de obra (procesos con un valor referencial mayor a 1800 000,00 soles) existe latente un alto riesgo al elegir al mejor postor ya que se requiere evitar incumplimientos durante la ejecución de las obras. Este riesgo es elevado aun en el caso de un empate, ya que el sorteo en acto público no asegura que la buena pro la obtenga el postor más conveniente para los intereses del Estado, siendo incierto el porcentaje de éxito de la ejecución del proyecto a futuro. A esto se suma la dificultad de que la legislación actual no está adecuada para que este tipo de proceso pueda realizarse en forma autómata, donde la buena pro se otorgue mediante un proceso virtual en el que las variables hayan sido reducidas a factores de diferenciación normalizados para otorgarla.

Es conveniente tener en cuenta que el riesgo es la probabilidad de tener éxito o fracaso, cuando se toma la decisión de llevar a cabo un proyecto (Del Carpio y Eyzaguirre, 2007). Se hace necesario realizar una evaluación del riesgo que asume una entidad del Estado cuando califica a los postores que concursan por adjudicarse la buena pro de un proceso, en términos de la probabilidad de haber elegido al mejor postor, es decir, aquel con mayor probabilidad de cumplimiento (éxito) en la ejecución del proyecto convocado. De este modo se pueden tomar las decisiones más adecuadas para salvaguardar los intereses del Estado.

El problema principal que enfrenta una entidad del Estado es poder calcular con el mínimo error posible cuál de los postores -empresas contratistas - que concursan por la buena pro tiene mayor probabilidad de tener éxito en la ejecución del proyecto convocado por la entidad, sobre 
todo si se da el caso de empate, en el cual tiene que dilucidarse el otorgamiento de la buena pro mediante un sorteo. Asimismo, es conveniente tener presente la probabilidad de éxito que tiene la empresa contratista ganadora de la buena pro antes de iniciar el proyecto, a fin de que la entidad pueda tomar decisiones con los resultados obtenidos.

Es posible que la entidad realice un cálculo objetivo y estime la probabilidad de éxito de cada postor en la ejecución del proyecto al cual concursa, con base en una evaluación de variables mediante la comparación de valores umbrales con proyectos similares ejecutados, calificando el proyecto antes de ser iniciado como éxito o fracaso. No obstante, el problema que puede darse es que los valores umbrales de comparación se determinan en condiciones de alta incertidumbre, ya que el cálculo se basa en una media ponderada de la exposición al riesgo usando datos históricos de la empresa. Por otro lado, el problema que puede ocurrir es que la empresa adopte valores teóricos de comparación (por ejemplo, valores pertenecientes a otras organizaciones), a fin predecir el éxito o fracaso de un proyecto. En ambos casos el proceso de estimación del riesgo se realiza con alta incertidumbre y no es confiable.

Para revertir la situación planteada, a continuación se enuncian las siguientes interrogantes:

¿Es posible que en el procedimiento de selección Adjudicación Simplificada se pueda generar un criterio de decisión autómata en caso de empate y que pueda tramitarse enteramente en forma virtual?

¿Es posible que en el procedimiento de selección Licitación Pública, en caso de empate, pueda elegirse al postor mediante un índice de cumplimiento pronosticado de acuerdo con el comportamiento de la empresa en la ejecución de proyectos de infraestructura similares?

¿Es posible que en el procedimiento de selección Licitación Pública este índice de cumplimiento pronostique el grado de probabilidad de éxito o fracaso de la obra a ejecutar?

¿Es posible predecir de manera confiable la probabilidad de cumplimiento que tiene una empresa contratista en la ejecución de un proyecto de infraestructura antes de ser iniciado?

¿Es posible construir un modelo basado en técnicas de inteligencia artificial que pueda calcular la probabilidad de cumplimiento (éxito o fracaso) de una empresa en la ejecución de un proyecto de infraestructura convocado por una entidad del Estado?

\section{Estrategia de solución}

Se plantea el diseño de un modelo referido a proyectos de ejecución de obras públicas a fin de proporcionar una arquitectura genérica que actúe en forma autónoma en el procedimiento de selección Adjudicación Simplificada. Esto permitirá que al ser el trámite comple- 
tamente virtual pueda generarse un criterio de decisión autómata en caso de empate, en el que se propone la elección del postor mediante un sorteo electrónico en un sistema de aleatoriedad controlada basada en el método aleatorio SHA512 de encriptación y la transformación del hash. Para el procedimiento de selección Licitación Pública, en caso de empate, se propone la elección del postor mediante un índice de eficiencia pronosticado por un sistema, de acuerdo al comportamiento de la empresa en la ejecución de proyectos de infraestructura similares. En este caso se genera un modelo que realiza la predicción de la probabilidad de éxito o fracaso del postor al ejecutar el proyecto antes de iniciarlo. Este proceso supone pronosticar el índice de eficiencia de cada uno de los postores que participan en un procedimiento de selección Licitación Pública para la contratación de la obra, el cual puede servir para realizar un desempate en caso de que algunos postores igualen en el puntaje final. También requiere pronosticar el índice de eficiencia del postor ganador de la buena pro en relación a su probabilidad de éxito o fracaso en el proyecto, realizando un replanteo de dichas probabilidades, comparando los datos históricos con el comportamiento del postor respecto de los riesgos identificados en el proceso de negocio y usando para tal fin las redes neuronales artificiales como herramienta de análisis.

Se propone la integración de información para concursos públicos mediante base de datos centralizada para facilitar el registro y selección. Esta base de datos debe contener la información de las experiencias del personal profesional clave, formación académica, experiencia del postor en obras similares y generales. De esta forma el proceso de selección de empresas se hará de manera automática usando sistemas autónomos que operen con dicha base de datos. La figura 3 muestra un resumen de nuestra propuesta.

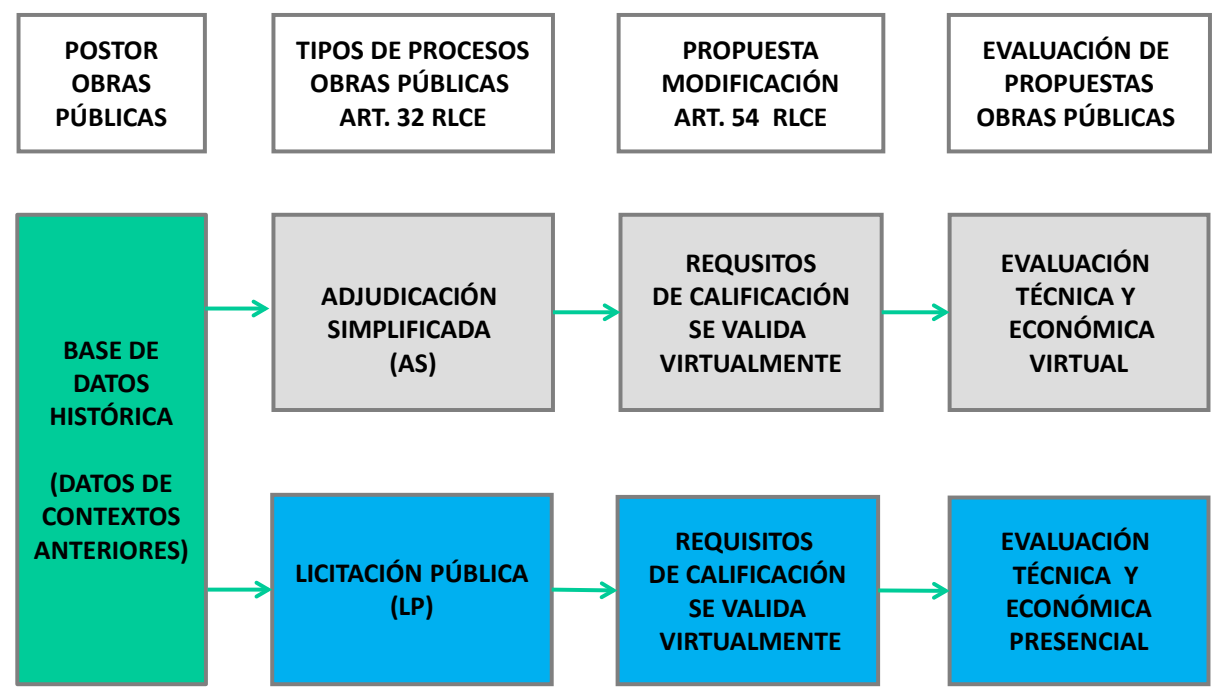

Figura 3. Propuesta respecto a validación virtual requisitos de calificación

Elaboración propia 


\section{Metodología de solución}

\subsection{Elección en caso de empate en el procedimiento de selección Adjudicación Simplificada}

La figura 4 propone una validación de requerimientos técnicos mínimos, con una base de registro y selección que construirá la empresa, de modo que pueda ser información registrada en los campos de la base de datos. Pare ello se plantea una modificación en el artículo 67 del RLCE en cuanto a la estandarización de los requisitos de calificación, como equipamiento estratégico, formación académica, experiencia del postor en obras similares y generales, que puedan exigir las entidades en el procedimiento de selección Adjudicación Simplificada. Se emplearán fichas técnicas de acuerdo a cada tipo de obra, de modo que se facilite el proceso de adjudicación de la buena pro en forma autónoma. La figura 4 muestra el proceso que se debe realizar en caso de empate.

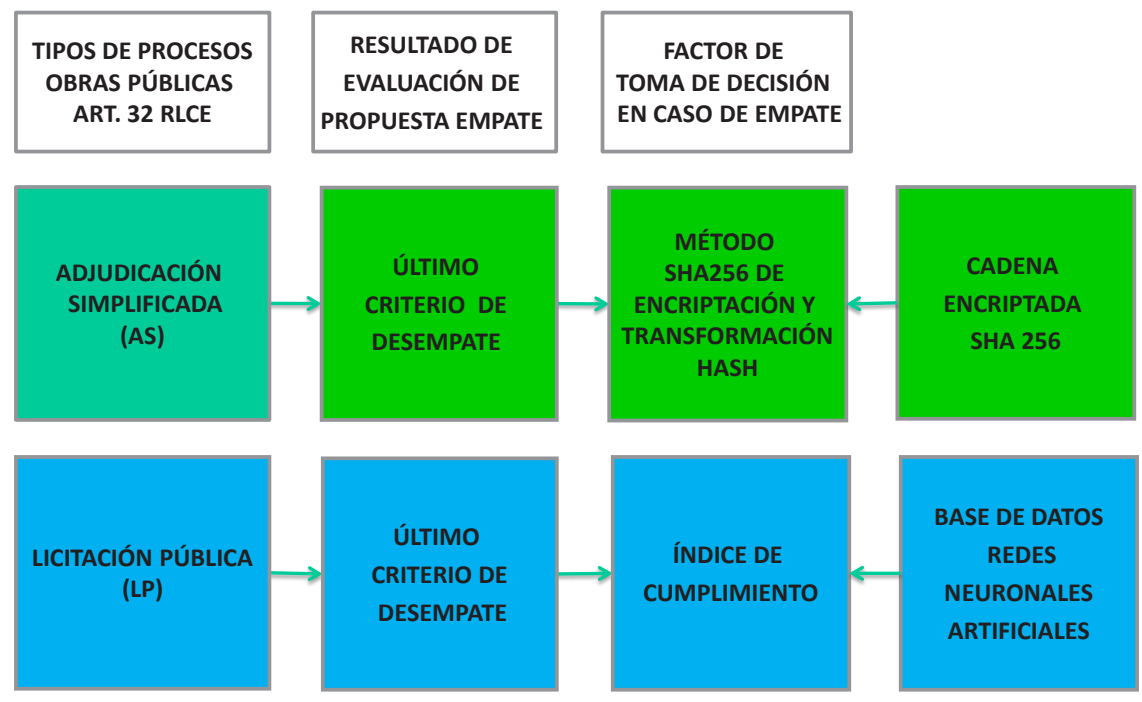

Figura 4. Proceso decisorio en caso de empate

Elaboración propia

Para el procedimiento de selección Adjudicación Simplificada, en caso de empate se propone la elección del postor mediante un sorteo electrónico mediante un sistema de aleatoriedad controlada basada en el método aleatorio SHA256 de encriptación y la transformación del hash.

Para seleccionar una empresa ganadora en caso de empate, se asigna una cadena de texto a cada postor con el siguiente formato: 
Nombre del Proyecto + RUC Empresa + RUC Empresa $1+$ RUC Empresa $2+$ RUC Empresa $3+$ + RUC Empresa X

Esta cadena asignada a cada postor luego es transformada con el método de encriptación SHA256 en una cadena de tamaño fijo única representativa de la cadena de entrada. La cadena encriptada resultante está en formato hexadecimal. Cada postor tiene asignada la cadena encriptada que le corresponde.

De la nueva cadena encriptada se escogen los ocho primeros caracteres y estos se transforman de hexadecimal a decimal, para ser divididos entre 42949672,95 que es $\left(2^{32}-1\right) / 100$ representativo al máximo valor decimal de ocho caracteres hexadecimales FFFFFFFF entre 100.

Ejemplo de aplicación y análisis técnico:

Proyecto: Parque Distrito X

RUC de la Empresa participante 1: 123456789

RUC de la Empresa participante 2: 321654987

RUC de la Empresa participante 3: 789456123

RUC de la Empresa participante 4: 123789456

En el caso de que empaten la empresa 1 y la empresa 3, cadena representativa de la empresa 1:

Parque Distrito X 123456789123456789321654987789456123123789456

Cadena representativa de la empresa 3:

Parque Distrito X 789456123123456789321654987789456123123789456

Después de pasar por la encriptación SHA256, cadena encriptada representativa de la empresa 1 en formato hexadecimal:

1A3B1F3203C61A1B01BB265288A86C768DA0CF9771582EE10947E823401EB06B

Cadena encriptada representativa de la empresa 3 en formato hexadecimal:

F7141523F6E076391A8FBE7DE505A3C2EF016DF9840198C4FAC93A2DFC095B30

Una vez aplicada la conversión a decimal y la división de los ocho primeros caracteres:

Empresa 1: 440082226 / $42949672,95=10,25$

Empresa 3: 4145288483 / $42949672,95=96,52$ 
La empresa 3 es la ganadora del proceso de selección. La figura 5 muestra el procedimiento de conversión detallado.

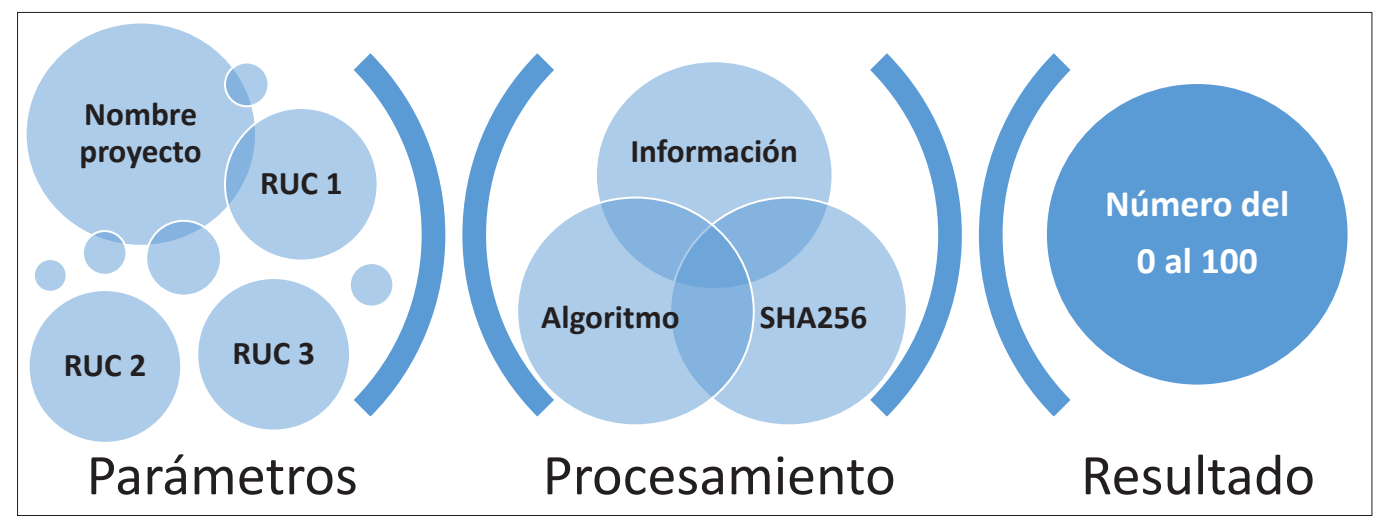

Figura 5. Proceso de encriptación

Fuente: Variante del Provably Fair implementada y explicada por bitzino.com

Al no tener el proceso de selección entradas modificables y al ser completamente automático, se puede dar a conocer el algoritmo para la obtención de resultados y este siempre devuelve el mismo resultado, siempre y cuando se introduzcan los mismos parámetros. De este modo, los postores pueden comprobar sus resultados con los parámetros que se utilizaron en la encriptación de la cadena incluso antes de que se realice el desempate en forma automática. Esta característica otorga transparencia al proceso. Para verificar el resultado lo que debe hacer el postor es evaluar sus parámetros con el algoritmo y comparar el resultado obtenido. Para esto la información del proyecto y los parámetros usados deben ser públicos. Los RUC de las empresas que participaron en el concurso del proyecto y el nombre del proyecto son la única información relevante para el algoritmo.

\subsection{Elección en caso de empate en el procedimiento de selección Licitación Pública (ADP)}

Un proceso más confiable, a diferencia del sorteo en caso de empate, es la selección del postor mediante un índice de cumplimiento pronosticado por un sistema, de acuerdo con el comportamiento de las empresas en la ejecución de proyectos de infraestructura similares. Nuestra propuesta consiste en establecer un modelo que utilice los datos históricos de proyectos similares ejecutados por la empresa, buscando identificar los factores de riesgo que afecten las variables de éxito del proyecto. El modelo propuesto usa como herramienta de análisis las redes neuronales artificiales para determinar los umbrales de comparación de éxito o fracaso de un proyecto antes de ser iniciado. 
El modelo propuesto será construido con base en los datos históricos de proyectos similares ejecutados por cada empresa postora, usando como herramienta las redes neuronales artificiales y tendrá como salida o variable de evaluación estimada el indicador del objetivo del proyecto (efectividad, eficiencia y calidad). El cálculo del riesgo se hará en términos de la probabilidad de éxito o fracaso del proyecto antes de ser iniciado, a lo que llamaremos el índice de cumplimiento de cada postor. Esta medida estimada del riesgo debe definir las estrategias y planes de monitoreo o mitigación de riesgos que podría emplear la entidad con los resultados obtenidos respecto de los postores y eventualmente del postor ganador.

La evaluación del riesgo constituye la información primaria para el proceso decisorio, por lo tanto nos encontramos con el problema de averiguar en qué medida los riesgos pueden repercutir en los objetivos del proyecto. Por ejemplo, si los riesgos impactan demasiado sobre el proyecto, debemos obtener baja probabilidad de éxito. Sin embargo, para evaluar los resultados debemos tener en cuenta la base o umbral de comparación que arroja el modelo. Por ejemplo, supóngase como objetivo del proyecto medir la efectividad como índice de cumplimiento (resultados alcanzados sobre resultados planificados) a fin de definir el éxito o fracaso de un proyecto para luego compararlo con los índices de cumplimiento de proyectos similares de la empresa. Supongamos que para un proyecto se obtiene un índice de cumplimiento IC 1 entre 0,7 y 0,8. Si se define un índice de cumplimiento teórico de éxito IC2 = 1,0 como umbral de comparación, entonces el proyecto es considerado como fracaso, porque IC1 < IC2. Por el contrario, si hemos elegido como umbral el valor que arroja el modelo basado en redes neuronales artificiales teniendo como dato la información de todos los proyectos similares ejecutados por la empresa (y esta fuera, IC3 $=0,65$ ) tendríamos un éxito (IC1 $>I C 3$ ). Esto último ocurre porque el objeto de comparación se basa en experiencias reales de la organización. Por lo tanto, debemos procurar calibrar el criterio de evaluación de los datos observados con base en las actuaciones reales de la empresa, procurando que la información sea suficiente y lo más actualizada posible, a fin de estimar un modelo óptimo que use la herramienta de las redes neuronales artificiales.

La estrategia que se propone está basada en el diseño de indicadores históricos comunes a los proyectos de una organización, que permitan construir una base de datos históricos del comportamiento de la empresa en la ejecución de proyectos similares, con el fin de calcular el impacto de los factores de riesgo sobre los objetivos del proyecto. El propósito de esta estrategia es generar una base de comparación del valor del riesgo.

Este documento propone definir los objetivos de un proyecto bajo un "esquema de cumplimiento" (Lezama, 2007). El esquema permite identificar los indicadores de cumplimiento relacionando elementos cuantificables para luego transformarlos en indicadores básicos. El esquema de cumplimiento está sostenido por tres indicadores 
generales: Efectividad (IPE), Eficiencia (IPP) y Calidad (IPC); el promedio de estos tres indicadores nos dará el índice de cumplimiento (IC). Este punto de vista se utilizará en el resto de este documento.

Con el fin de comprobar si un proyecto está cumpliendo sus objetivos declarados, consideramos los umbrales de efectividad, eficiencia y calidad. De esta manera, el cumplimiento -éxito o fracaso del proyecto- se define según la forma en que se establecen estos umbrales. Esta interpretación nos permite definir un proyecto como exitoso sobre la base de un aspecto específico (por ejemplo, un proyecto puede considerarse exitoso si el factor de cumplimiento supera o iguala el umbral establecido por el modelo; en caso contrario el proyecto puede considerarse un fracaso). Sin embargo, este concepto puede ser representado por una función de muchas variables que calcula la métrica que hemos elegido para representar el éxito. Asimismo, usando la herramienta de redes neuronales artificiales, podemos calcular la probabilidad de cumplimiento (éxito) para el proyecto actual. Si esta probabilidad es inferior al umbral escogido, el impacto de los factores de riesgo aumenta y tiene que activarse un plan de contingencia para mitigarlo; en caso contrario, si esta probabilidad es superior al umbral definido, el impacto de los factores de riesgo disminuye y, por lo tanto, la influencia de los factores de riesgo sobre los objetivos del proyecto (esquema de cumplimiento) es mínima.

Los datos históricos basados en los factores de riesgo permitirán construir una función de regresión para la evaluación del riesgo ya que permiten generar como salida los umbrales de comparación de los objetivos del proyecto. Esta función es generada usando como herramienta las redes neuronales artificiales, cuyo resultado representa la métrica que hemos elegido para representar el éxito.

La figura 6 describe el impacto de los factores de riesgo sobre los objetivos del proyecto con base en los datos históricos de la organización. Sin embargo, debemos definir los indicadores adecuados que permitan medir si los objetivos de un proyecto (efectividad, eficiencia y calidad) son alcanzados mediante la comparación de umbrales a los que denotaremos como métricas de éxito o indicadores de cumplimiento. Asimismo, se deben definir los indicadores que miden los factores de riesgo y que impactan sobre los objetivos del proyecto, a los que denotaremos como indicadores de factores de riesgo. 


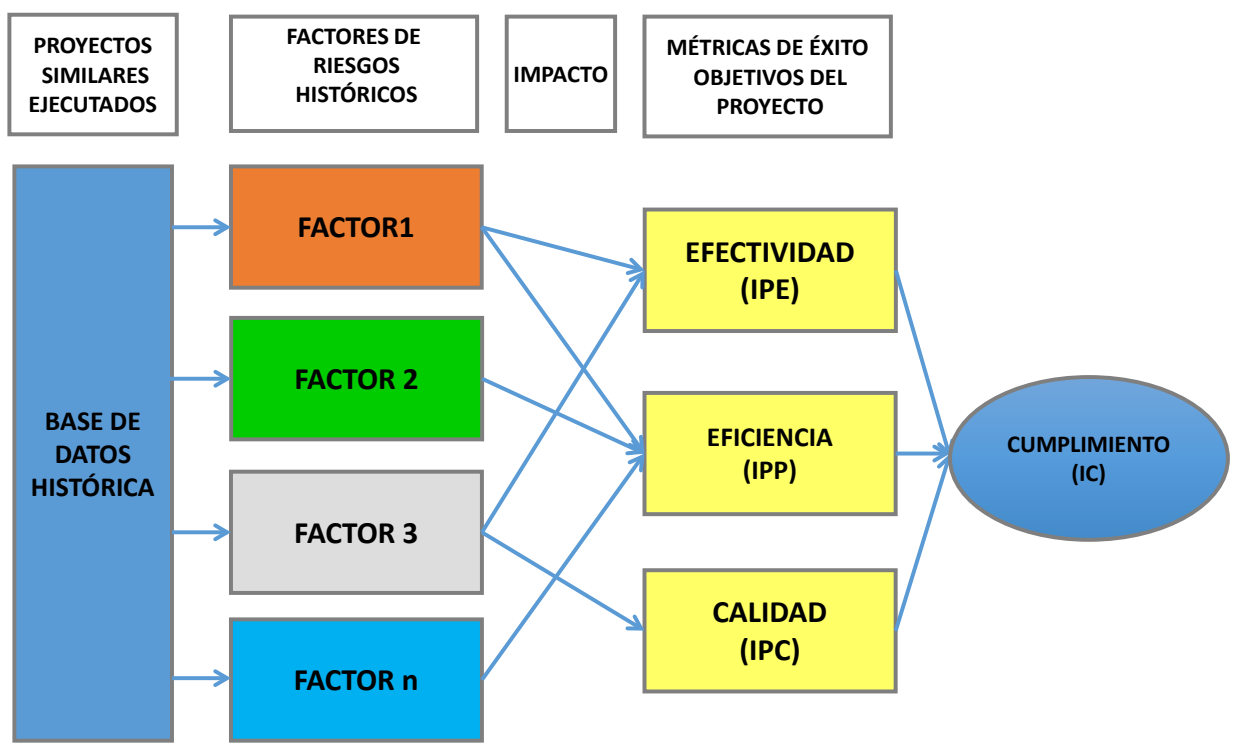

Figura 6. Base de datos histórica de proyectos similares ejecutados

Elaboración propia

\subsubsection{Selección del proyecto}

En este documento la metodología de solución propuesta identifica los indicadores adecuados que permitan calcular con el mínimo error posible la probabilidad de cumplimiento (éxito o fracaso) que tiene un postor en la ejecución del proyecto de infraestructura al cual postula, a fin de que la entidad estatal que licita el proyecto pueda usar este índice en caso de empate o tomar las decisiones que correspondan en resguardo de los intereses del Estado, ya sea confirmando las predicciones de éxito o fracaso del proyecto, o replanteándolas. Asimismo, los datos históricos basados en los factores de riesgo permiten generar como salida los umbrales de comparación de los objetivos del proyecto, usando como herramienta las redes neuronales artificiales, cuyo resultado representa la métrica que hemos elegido para representar el cumplimiento del proyecto (éxito).

Un ejemplo de empresas que ejecutan proyectos recurrentes son las empresas constructoras que llevan a cabo proyectos de infraestructura con el Estado, en los cuales podemos definir los indicadores de objetivos y los indicadores de los factores de riesgo, los cuales nos permitirán calcular con el mínimo error posible la probabilidad de cumplimiento (éxito o fracaso) que tiene el postor en la ejecución del proyecto que se licita, mediante la técnica de inteligencia artificial: redes neuronales artificiales.

A continuación se definen los indicadores de objetivos y los indicadores de factores de riesgo para el caso de una empresa constructora de obras de 
infraestructura, tomando como referencia el modelo de gestión de indicadores de Salgueiro (2001).

\subsubsection{Indicadores de objetivos}

En el apartado 5.3 se definieron los objetivos de un proyecto bajo un "esquema de cumplimiento" (figura 6). El esquema permite identificar los indicadores que aseguren el cumplimiento de una empresa contratista en la ejecución de un proyecto de infraestructura que licita el Estado. El esquema de cumplimiento propuesto está sostenido por tres indicadores generales: efectividad, eficiencia y calidad.

A continuación identificamos los indicadores de cumplimiento de objetivos que son medidas de éxito o fracaso en relación a los objetivos de un proyecto, a los cuales denominaremos métricas de éxito del proyecto. Los indicadores son definidos para el caso de una empresa constructora de obras de infraestructura tomando como referencia el modelo de gestión de indicadores de Salgueiro (2001). Los indicadores objetivos de cumplimiento están sostenidos por los tres indicadores generales que ya se han mencionado: IPE, IPP e IPC; el promedio de estos tres indicadores nos dará el índice de cumplimiento IC.

\section{Indicador de efectividad}

Este indicador proporciona información cuantificable sobre el cumplimiento del objetivo planteado respecto a las penalidades contractuales aplicadas al ejecutor de obras, y representa un índice del cumplimiento de la prestación de ejecución de obra. Se propone el indicador denominado efectividad en la ejecución de la prestación sin penalidades (IPE). Lo definiremos como el grado de cumplimiento del plan de presupuesto de la prestación de ejecución de obra sin penalidades contractuales, por la variable IPE.

$\frac{\text { PENALIDAD MÁXIMA-PENALIDADES APLICADAS }}{\text { PENALIDAD MÁXIMA }}$

\section{Indicadores de eficiencia}

Representan la capacidad del proyecto de lograr su conclusión en el mínimo tiempo y con el menor costo unitario posible (Lezama, 2007). En este sentido se propone un indicador de eficiencia denominado relación del plazo de ejecución del proyecto (IPP), el cual indica la capacidad de concluir el proyecto en el mínimo tiempo posible.

$$
\frac{\text { PLAZO PROGRAMADO DE EJECUCIÓN }}{\text { PLAZO REAL DE EJECUCIÓN }}
$$




\section{Indicadores de calidad}

Representan la capacidad del proyecto para satisfacer al cliente y cuantifican la mejora continua de los procesos y resultados. El indicador de calidad propuesto es calidad del proceso (IPC), el cual mide precisamente la calidad del proceso (Apaza, 2003). Es una relación entre el avance de ejecución de obra aprobado por la entidad (metrado ejecutado conforme) y el avance de ejecución de obra realmente ejecutado (metrado ejecutado real).

METRADO DE EJECUCIÓN CONFORME

METRADO DE EJECUCIÓN REAL

\subsubsection{Indicadores de los factores de riesgo}

Durante la evaluación del riesgo y el diseño del proceso de toma de decisiones, debemos identificar los eventos que podrían impedir, degradar, demorar o mejorar el logro de los objetivos de la empresa. Estos eventos constituyen las fuentes externas o internas de riesgo en relación a los objetivos de la empresa, que en adelante denominaremos factores de riesgo.

Se debe tener especial cuidado al definir los factores de riesgo, procurando identificar factores característicos del negocio, de modo que estos no resulten teóricos, irreales o de medición complicada. Se debe lograr que los datos sean lo más exactos posible y que puedan ser obtenidos con facilidad. Se deben identificar los factores de riesgo que tienen impacto positivo o negativo en los objetivos de un proyecto de infraestructura.

Los factores de riesgo que afectan o impactan la medición de las métricas de éxito del proyecto deben ser expresados por indicadores a calcularse para todo proyecto de infraestructura. Estos indicadores son las entradas al modelo propuesto de la figura 6, que tiene como salida la métrica de éxito del proyecto y que será expresada en una función de regresión no lineal. El modelo requiere un gran número de indicadores de factores de riesgo, para obtener buenos resultados; sin embargo, si el número de indicadores crece, la métrica de éxito del proyecto será más difícil de estimar utilizando la estadística clásica.

Una solución a este problema es usar la herramienta de redes neuronales artificiales, en particular el modelo multicapa feed-forward backpropagation, para calcular los valores de las funciones de regresión, de modo que el número de factores considerados no influye sobre el procedimiento. Este documento toma 
como referencia el modelo de validación de eficiencia adoptado por Cantone, Sarciá y Basili (2007), basado en el análisis de redes neuronales artificiales como un medio para estimar una función de regresión no lineal, incluso si el número de factores de riesgo es elevado.

Para identificar los factores de riesgo que afectan los objetivos del proyecto hemos tomado como referencia, entre otros, el modelo Goal Question Metric (Basili, Caldiera, Rombach, 1994).

Si bien este modelo fue creado para estimaciones en proyectos de software, el enfoque Goal Question Metric proporciona un método eficaz para identificar factores e indicadores tanto del proceso como de los resultados de proyectos de infraestructura, considerando que un programa de medición puede ser más satisfactorio si es diseñado teniendo en cuenta los objetivos perseguidos. En este enfoque las preguntas potencialmente medibles ayudan a establecer si se está alcanzando en forma exitosa la meta trazada. Algunas preguntas posibles son las siguientes: ¿Qué factores repercuten en el logro del objetivo del proyecto? ¿Cuál es el contexto del proyecto? ¿Cuál es el contexto de la organización?

La tabla 1 contiene nuestra propuesta de los factores de riesgo para proyectos de infraestructura y definición de indicadores del riesgo para medir el impacto sobre los objetivos (métricas de éxito) del proyecto. La escala de medición se elaboró con base en los datos provenientes de las experiencias de proyectos similares ejecutados (valores máximos y mínimos) por empresas ejecutoras de obras y de los valores límite permitidos por la legislación vigente.

Tabla 1. Factores de riesgo e indicadores de impacto

\begin{tabular}{|l|l|c|c|c|}
\hline \multicolumn{1}{|c|}{$\begin{array}{c}\text { Factores } \\
\text { riesgo }\end{array}$} & \multicolumn{1}{|c|}{ Indicador } & Medición & Símbolo & $\begin{array}{c}\text { Escala } \\
\text { de medición }\end{array}$ \\
\hline Precio ofertado del proyecto & $\begin{array}{l}\text { Relación presupuesto ofertado y } \\
\text { presupuesto base. }\end{array}$ & $\frac{\text { PRESUPUESTO OFERTADO }}{\text { PRESUPUESTO BASE }}[0,9 ; 1,10]$ \\
\hline Gastos generales del proyecto & $\begin{array}{l}\text { Relación gastos generales totales } \\
\text { (fijos más variables) y costo directo } \\
\text { de la obra (presupuesto contratado } \\
\text { menos impuestos, gastos generales } \\
\text { y utilidades) }\end{array}$ & $\frac{\text { GASTOS GENERALES }}{\text { COSTO DIRECTO DEL PROYECTO }}$ & RGP & {$[0,0 ; 0,5]$} \\
\hline Utilidad prevista del proyecto & $\begin{array}{l}\text { Relación utilidad y costo directo de } \\
\text { la obra (presupuesto contratado } \\
\text { menos impuestos, gastos generales } \\
\text { yutilidades) }\end{array}$ & COSTO DIRECTO DEL PROYECTO & RUP & {$[0,0 ; 1,0]$} \\
\hline
\end{tabular}


(continuación)

\begin{tabular}{|c|c|c|c|c|}
\hline Tipo de proyecto & $\begin{array}{l}\text { Tipo de proyecto: } \\
\text { 1. Proyecto de edificación. } \\
\text { 2. Proyecto de saneamiento. } \\
\text { 3. Proyecto energético. } \\
\text { 4. Proyecto de irrigación. } \\
\text { 5. Proyecto de carreteras y puentes. }\end{array}$ & TIPO DE PROYECTO & TP & {$[1,2,3,4,5]$} \\
\hline Duración del proyecto & Plazo de ejecución del proyecto. & DÍAS & PE & {$[0 ; 1000]$} \\
\hline Experiencia en obras & $\begin{array}{l}\text { Experiencia del ingeniero residente } \\
\text { de obras. }\end{array}$ & AÑOS & EIR & {$[1 ; 40]$} \\
\hline Experiencia en seguridad & Experiencia del ingeniero de seguridad. & AÑOS & EIS & {$[0 ; 40]$} \\
\hline Experiencia en calidad & Experiencia del ingeniero de calidad. & AÑOS & EIC & {$[0 ; 40]$} \\
\hline Experiencia en administración & $\begin{array}{l}\text { Experiencia de la gerencia de } \\
\text { administración }\end{array}$ & AÑOS & EGA & {$[1 ; 40]$} \\
\hline $\begin{array}{l}\text { Variación de precios de mate- } \\
\text { riales, equipos, mano de obra } \\
\text { del sector construcción por } \\
\text { inflación }\end{array}$ & $\begin{array}{l}\text { Factor de reajuste K. Proviene de la } \\
\text { fórmula polinómica del proyecto y } \\
\text { es calculado con base en los índices } \\
\text { de precios unificados del sector cons- } \\
\text { trucción publicados por el Instituto } \\
\text { Nacional de Estadística e Informática } \\
\text { (INEI). }\end{array}$ & FÓRMULA POLINÓMICA DEL PROYECTO & K & {$[0.5 ; 2]$} \\
\hline
\end{tabular}

Elaboración propia

\subsubsection{Modelo de predicción de la métrica de éxito}

El modelo de predicción tiene como finalidad la estimación de la métrica de éxito del proyecto representado por una función de regresión no lineal. Para tal fin se utiliza una herramienta de redes neuronales artificiales. La métrica de éxito elegida es el índice de cumplimiento descrito en el apartado 5.3.2 y la estructura de la red neuronal artificial utilizada en el modelo es la de retropropagación conocida como red multicapa feed forward (Rumelhart, Hinton, Williams, 1986), donde las variables de entrada son los indicadores de los factores de riesgo del proyecto y la salida es la métrica de éxito del proyecto (estimación de función de regresión no lineal). Una descripción de este modelo se aprecia en la figura 6 , donde el modelo de predicción calcula la métrica de éxito del proyecto, dando como resultado el valor del IC.

En síntesis, el modelo permite predecir el IC del postor participante del proyecto licitado por una entidad del Estado (proyecto nuevo). Asimismo, sirve para generar una base de datos donde estén clasificados los proyectos ejecutados por la empresa como éxito o fracaso. En particular, si el IC real para un proyecto es mayor que el IC estimado por el modelo de predicción (valor umbral estimado por la red neuronal) el proyecto se clasifica como un éxito (1); en caso contrario se clasifica como un 
fracaso (0). Este dato se almacena en la base de datos con los indicadores de los factores de riesgo que le corresponden.

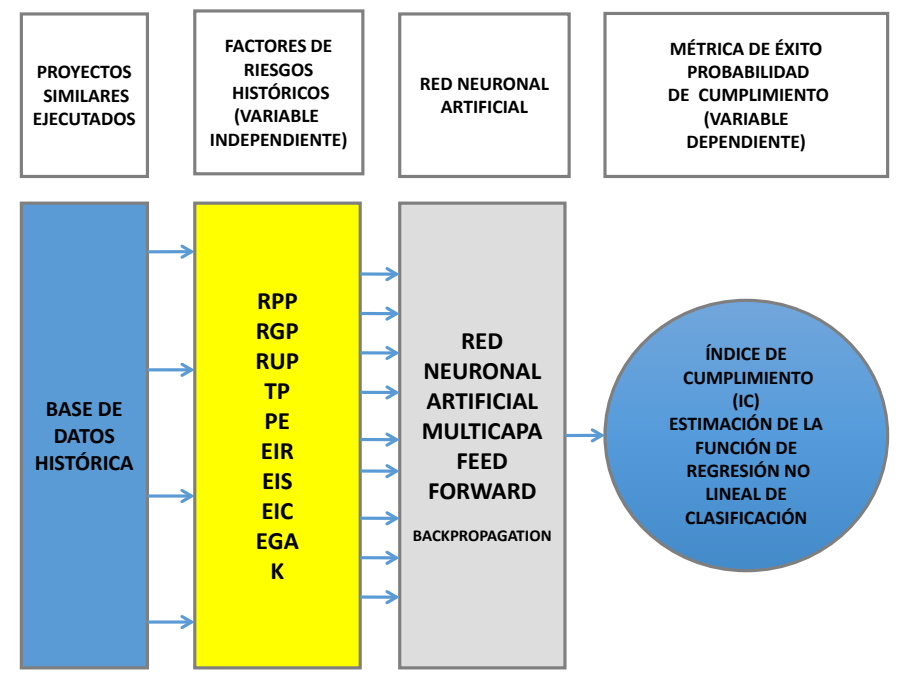

Figura 7. Modelo de predicción de la métrica de éxito

Elaboración propia

\subsubsection{Modelo de predicción de éxito o fracaso}

Una vez clasificados los proyectos ejecutados por la empresa como éxito (1) y fracaso (0), agrupados en forma conjunta con los indicadores de los factores de riesgo que determinan su clasificación, es posible construir un modelo que pueda predecir la probabilidad de cumplimiento (riesgo) de un nuevo proyecto, indicando su tendencia de cumplimiento (éxito o fracaso). Esta metodología implica el uso de una segunda red neuronal artificial feed forward donde las entradas son los indicadores de los factores de riesgo que afectan los objetivos del proyecto y la salida es un valor entre uno y cero que representa la probabilidad de cumplimiento (riesgo) de un nuevo proyecto. Para esto se establece un patrón de aceptación o rechazo (fijado en 0,5 o más) a fin de generar la probabilidad de cumplimiento del proyecto.

En este caso, si el valor de salida es de entre 0,5 y 1, se establece la tendencia de cumplimiento (éxito) del proyecto, y si el valor de salida es de entre 0 y menos de 0,5, se establece la tendencia al incumplimiento (fracaso) del proyecto. Una descripción de este modelo se aprecia en la figura 7. 


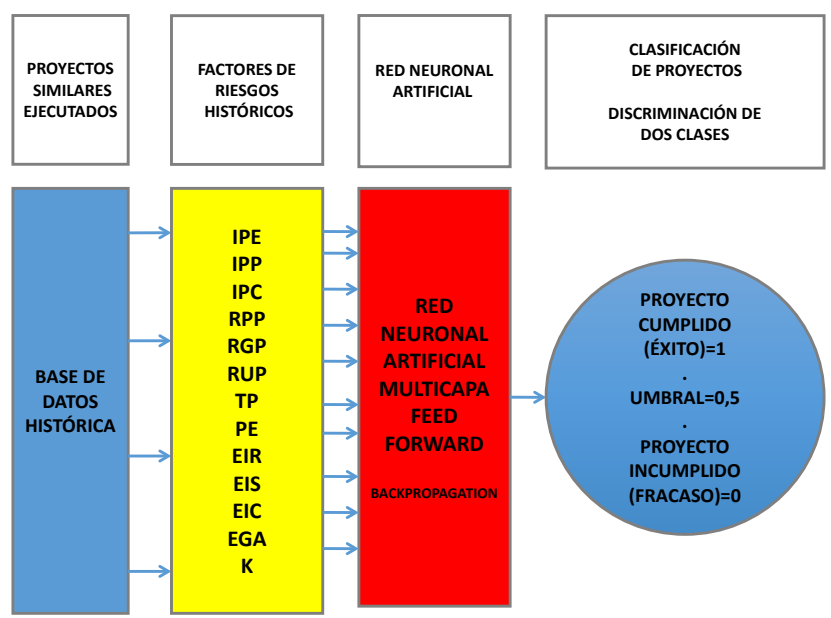

Figura 8. Predicción de la probabilidad de éxito o fracaso

Elaboración propia

\section{Caso de estudio}

\subsection{Interfaz de evaluación para el desempate}

La figura 9 muestra el procedimiento de conversión detallado.

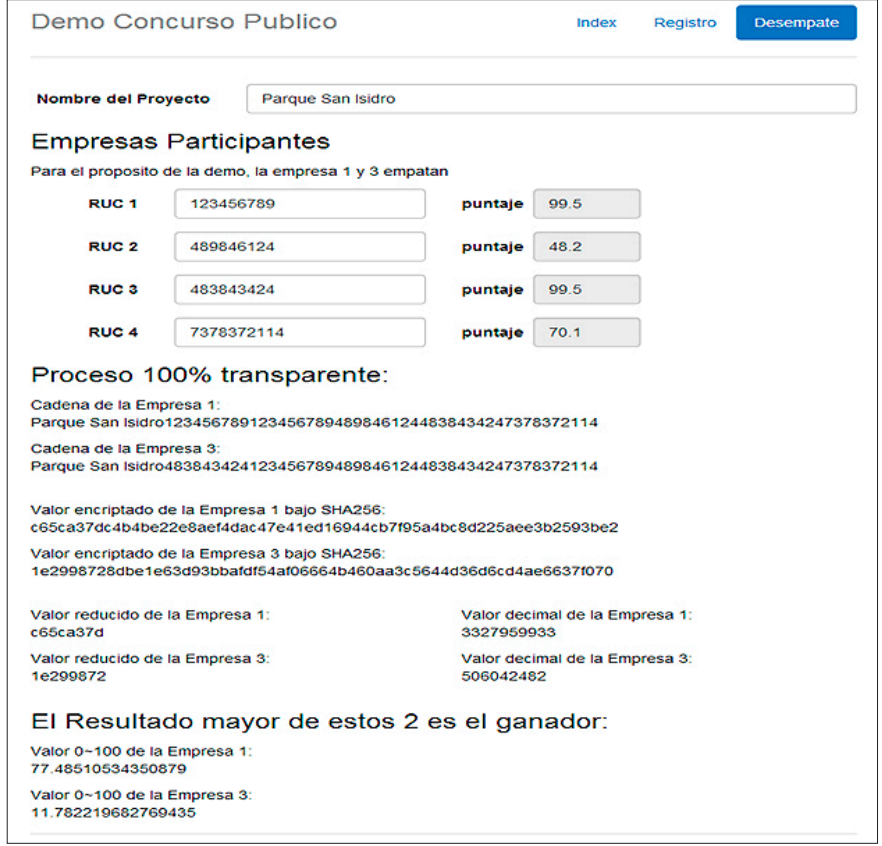

Figura 9. Predicción de la probabilidad de éxito o fracaso

Fuente: pantalla de la aplicación de prueba de concepto 
La interfaz HTML se hizo con la ayuda de la librería de estilos bootstrap y el MVC AngularJS. La funcionalidad del algoritmo se hizo con la ayuda de la implementación en javascript del algoritmo SHA256 escrito por Chis Veness. En la demo se simula un empate entre la empresa 1 y la empresa 3 arbitrariamente para poder demostrar el proceso de desempate.

El HTML de la interfaz:

$<$ html ng-app lang=»en» $><$ head $>$

$<$ meta charset=»utf-8»>

$<$ meta http-equiv=»X-UA-Compatible» content=»|E=edge»>

$<$ meta name=»viewport» content=»width=device-width, initial-scale=1.0»>

$<$ meta name=»description» content=»»>

$<$ meta name=»author» content=»»»>

$<$ title $>$ Investigación - Desempate $</$ title $>$

$<$ link href=»css/bootstrap.css» rel=»stylesheet»>

<link href=»css/narrow-jumbotron.css» rel=»stylesheet»>

<script src=»https://ajax.googleapis.com/ajax/libs/angularjs/1.2.10/angular.min.js»></ script $>$

<script type=»text/javascript» src=»js/desempate.js»></script $>$

$<$ style type=»text/css» $></$ style $></$ head $>$

$<$ body ng-controller=»TodoCtrl»>

$<$ div class=»container $\gg>$

$<$ div class=»header»>

$<$ ul class=»nav nav-pills pull-right» $>$

$<\mathbf{l i}><\mathbf{a}$ href=»index.htm|»>|ndex $</ \mathbf{a}></ \mathbf{l i}>$

$<\mathbf{l i}><$ a href=»registro.htm|» $>$ Registro $</ \mathbf{a}></ \mathbf{l i}>$

$<$ li class=»active» $><$ a href=»desempate.htm|» $>$ Desempate $</$ a $></$ li $>$

$</$ ul $>$

$<\mathbf{h} \mathbf{3}$ class=»text-muted» $>$ Demo Concurso Publico $</ \mathbf{h} \mathbf{3}>$

$</$ div $>$

$<$ form class=»form-horizontal» role=»form» >

$<$ div class=»form-group» $>$

$<$ label class=»col-sm-3 control-label»>Nombre del Proyecto $</$ label $>$

$<\operatorname{div}$ class=»col-sm-9»>

$<$ input type=»text» class=»form-control» placeholder=»Nombre del Proyecto»ng-model=»Proyecto»>

$</$ div $>$

$</$ div $>$

$<\mathbf{h} \mathbf{3}>$ Empresas Participantes $</ \mathbf{h} \mathbf{3}>$

$<$ p $>$ Para el proposito de la demo, la empresa 1 y 3 empatan $</$ p $>$

$<$ div class=»form-group»>

$<$ label class=»col-sm-2 control-label»>RUC $1<$ /label $>$

$<\operatorname{div}$ class $=»$ col-sm-4»> 
$<$ input type=»text» class=»form-control» placeholder=»RUC de la empresa 1»ng-model=»Empresa1»> $</$ div $>$

$<$ label class=»col-sm-1 control-label»>puntaje</label $>$

$<$ div class $=»$ col-sm-2»>

<input type=»text» class=»form-control» placeholder=»puntaje» value=99.5 disabled $>$

$</$ div $>$

$</$ div $>$

$<$ div class=»form-group» $>$

$<$ label class=»col-sm-2 control-label»>RUC $2<$ /label $>$

$<$ div class=»col-sm-4»>

$<$ input type=»text» class=»form-control» placeholder=»RUC de la empresa 2»ng-model=»Empresa2»> $</$ div $>$

$<$ label class=»col-sm-1 control-label»>puntaje</label $>$

$<$ div class $=»$ col-sm-2»>

$<$ input type=»text» class=»form-control» placeholder=»puntaje» value $=48.2$ disabled $>$

$</$ div $>$

$</$ div $>$

$<$ div class=»form-group»>

$<$ label class=»col-sm-2 control-label»>RUC $3<$ /label $>$

$<$ div class $=»$ col-sm-4»>

$<$ input type=»text» class=»form-control» placeholder=»RUC de la empresa 3»ng-model=»Empresa3»> $</$ div $>$

$<$ label class=»col-sm-1 control-label»>puntaje</label $>$

$<$ div class=»col-sm-2»>

$<$ input type=»text» class=»form-control» placeholder=»puntaje» value=99.5 disabled $>$

$</$ div $>$

$</$ div $>$

$<$ div class=»form-group»>

$<$ label class=»col-sm-2 control-label»>RUC $4<$ /label $>$

$<$ div class=»col-sm-4»>

$\langle$ input type=»text» class=»form-control» placeholder=»RUC de la empresa 4»ng-model=»Empresa4»> $</$ div $>$

$<$ label class=»col-sm-1 control-label»>puntaje</label $>$

$<$ div class $=»$ col-sm-2»>

$<$ input type=»text» class=»form-control» placeholder=»puntaje» value $=70.1$ disabled $>$

$</$ div $>$

$</$ div $>$

$<\mathbf{h} 3>$ Proceso $100 \%$ transparente: $</ \mathbf{h} \mathbf{3}>$

$<\mathbf{p}>$ Cadena de la Empresa 1: $<\mathbf{b r}>\{\{$ e1 seed() $\}\}</ \mathbf{p}>$

$\langle\mathbf{p}\rangle$ Cadena de la Empresa 3: $<\mathbf{b r}>\{\{$ e3seed()\}\} $</ \mathbf{p}>$

$<$ br $>$ 


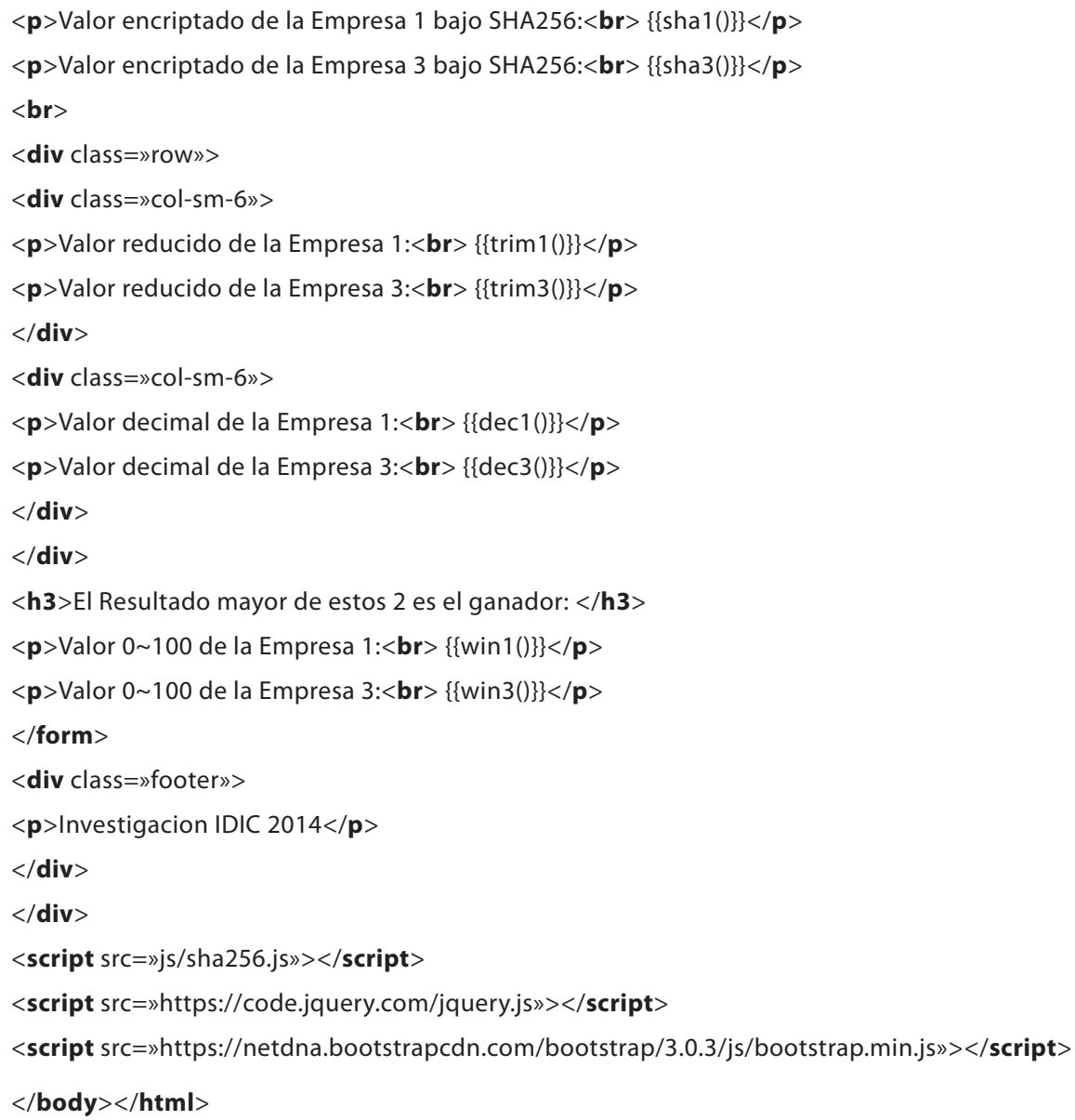

La interfaz del simulador usa AngularJS para mostrar los resultados en tiempo real, demostrando que los cálculos se pueden hacer del lado del cliente usando solamente la información mostrada en esta, así como los pasos para llegar al resultado final. Para el propósito de la demo solo se evalúan las puntuaciones de las empresas 1 y 3 , las cuales empatamos con un puntaje de 99,5.

El Javascript ejecutando la evaluación:

function TodoCtrl(\$scope) \{

//inicializamos valores arbitrarios solo para la demo (pueden ser modificados haciendo uso de la interfaz)

\$scope.Empresa1="123456789";

\$scope.Empresa2="489846124";

\$scope.Empresa3="483843424";

\$scope.Empresa4="7378372114";

\$scope.Proyecto="Parque San Isidro" 


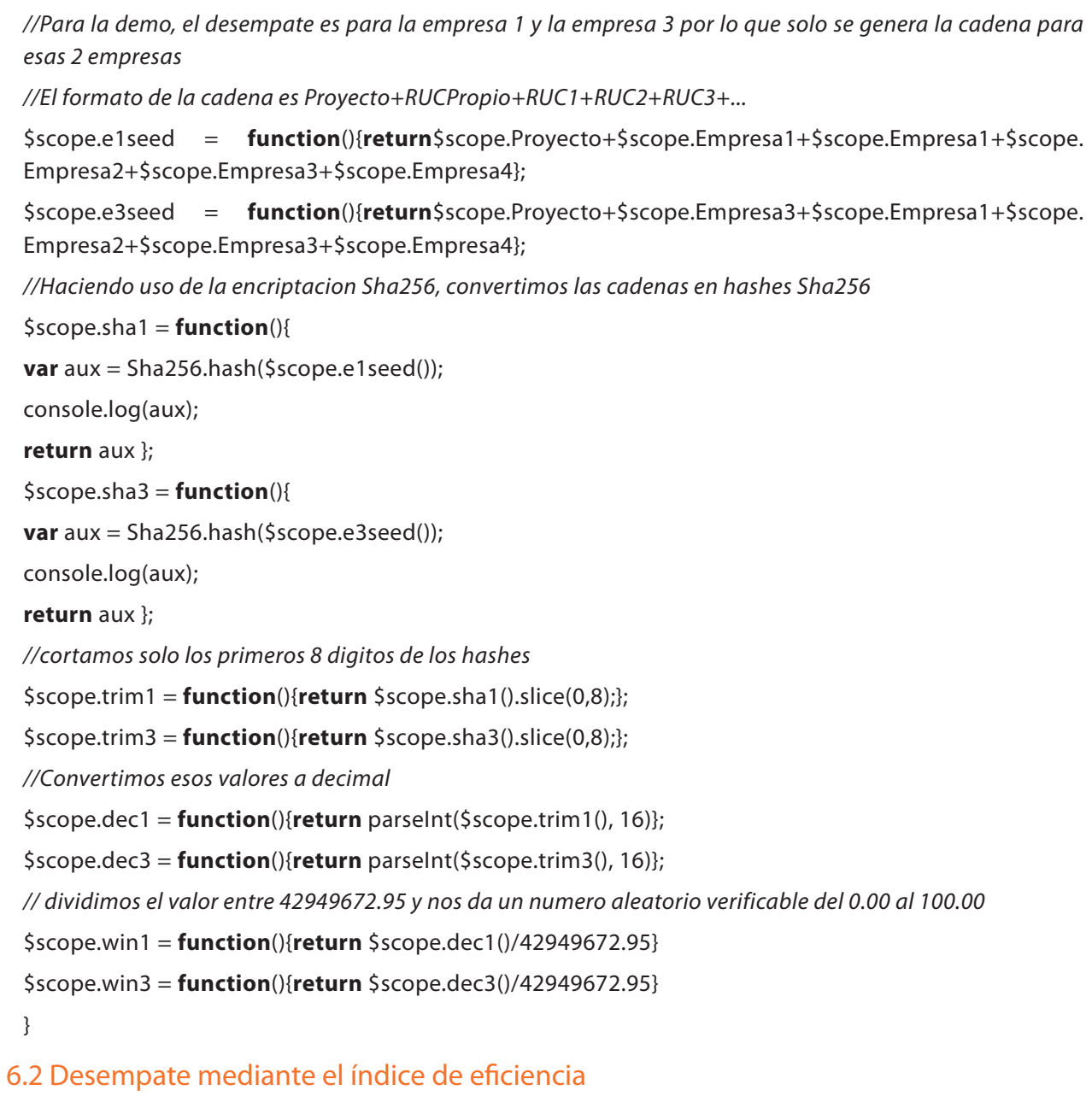

En este caso se considera un conjunto real de observaciones conformado por la información de los proyectos ejecutados por un grupo empresarial peruano formado por cinco empresas dedicadas a la ejecución de obras públicas. Se logró contar con la data histórica de 209 proyectos de construcción ejecutados por el grupo empresarial desde 1988.

En cada proyecto se identificaron 11 indicadores haciendo un total de 2299 datos que representan los índices de los factores de riesgo diseñados en el apartado 5.3.2 del presente documento. Los indicadores usados para medir los factores de riesgo que afectan a los objetivos de cada proyecto se presentaron en la tabla 1 del apartado 5.3.3.

\subsubsection{Elección del modelo}

Las pruebas de clasificación se efectuaron con el modelo de red neuronal artificial multicapa feed forward (Rumelhart, Hinton y Williams, 1986) usando el algoritmo 
de aprendizaje de retropropagación de Levenberg-Marquardt (gradiente descendente optimizado). A fin de mejorar la precisión de la red, se empleó una cantidad más amplia de datos, considerando un conjunto de prueba adicional al de validación. Se probaron 16 arquitecturas de redes neuronales artificiales. La distribución del número de capas, como la cantidad de neuronas por capa, se dio bajo la permutación de los números 5 , 9, 10 y 20 (obtenidos por una función random de rango [2; 20]). El programa utilizado para la simulación y entrenamiento de la red fue MatLab (Neural Networks Toolbox); asimismo se empleó el software Minitab Solutions para el cálculo de los datos estadísticos y la elaboración de histogramas. La función de transición empleada en la red perceptrón multicapa feed forward es la función sigmoidal para la capa de entrada y las capas ocultas, y una función lineal para la capa de salida de la red.

De los 209 conjuntos de datos empleados (cada conjunto tiene 11 indicadores) en la preparación de las redes, se emplearon 171 en el conjunto de entrenamiento, 19 en el conjunto de validación y 19 en el de prueba.

\subsubsection{Evaluación de la red}

El entrenamiento de las diferentes arquitecturas de redes neuronales artificiales se evaluó teniendo en consideración dos métricas: el grado de error y el rendimiento (Mse). Para la medición del grado del error de cada arquitectura se empleó el error absoluto medio (mean) del conjunto de validación y del conjunto de prueba. Finalmente, la definición del mejor modelo se realizó con base en el cálculo del error absoluto medio (mean) de ambos conjuntos. Para la evaluación de los resultados de salida de las diferentes arquitecturas probadas, se empleó el índice de rendimiento (Mse) proporcionado por MatLab (error cuadrático medio relativo al conjunto de entrenamiento) y la media del error absoluto (mean) de los conjuntos de validación y prueba. Además, se calculó la desviación estándar del error absoluto de ambos conjuntos para observar la estabilidad de la red. Los resultados mostrados indican que la arquitectura que presenta menor error y que mejor se ajusta para la predicción del modelo es la red feed forward $20 \times 10$ (20 capas ocultas, 10 neuronas en la capa escondida, 10 neuronas en la entrada y una neurona en la salida). Por lo tanto, se escogió la mencionada red como la más apta para la predicción del modelo debido al bajo porcentaje de error que obtiene en los conjuntos de validación y prueba. Se descarta el índice del rendimiento pues es un índice referencial al entrenamiento y a la validación (Matlab solo emplea el conjunto de la prueba para la visualización gráfica de los resultados de la regresión).

\subsubsection{Clasificación de los proyectos en dos clases: éxito y fracaso}

Se procedió a clasificar los proyectos históricos de la empresa (proyectos ejecutados) en un conjunto de dos clases: éxito (1) o fracaso (0), mediante la comparación del 
valor que arroja la red: el valor umbral del índice de cumplimiento (IC), el cual es comparado con el IC real del proyecto ejecutado. Si el IC real es mayor o igual que el IC que arroja el modelo de predicción, entonces lo clasificamos como éxito; en caso contrario, como fracaso. Considerando este esquema se puede construir una base de datos histórica donde los proyectos de infraestructura ejecutados por la empresa forma una clase de proyectos cumplidos (éxito) con clasificación 1 y una clase de proyectos incumplidos (fracaso) con clasificación 0. Las salidas se determinaron según el signo de la diferencia entre el valor real y el valor estimado del índice IC; en caso de ser no nulo o positivo se califica el valor como 1 (éxito), en caso de ser negativo (si realmente está por debajo del promedio) se califica como fracaso.

\subsubsection{Cálculo de la probabilidad de éxito o fracaso de un proyecto}

Una vez construida la base de datos que contiene todos los proyectos ejecutados por la empresa, con sus indicadores de los factores de riesgo y su respectiva clasificación como éxito (1) o fracaso (0), se procedió a implementar el modelo que prediga la probabilidad de cumplimiento de un nuevo proyecto, indicando su tendencia de cumplimiento (éxito) o incumplimiento (fracaso). Esta metodología implicó el uso de una segunda red neuronal artificial feed forward donde las entradas son los indicadores de los factores de riesgo que afectan los objetivos del proyecto, y la salida que se obtuvo es un valor entre uno y cero que representa la probabilidad de riesgo de un nuevo proyecto. Por ejemplo, un valor de salida 1 indicará que el proyecto será un éxito y un valor 0 significará que el proyecto será un fracaso. Si la salida es un valor fraccionario como 0,3 significa que existe un $30 \%$ de probabilidad de éxito del proyecto y un $70 \%$ de probabilidad de que los riesgos se produzcan. $\mathrm{Si}$ la salida es un valor de 0,9 indicará que existe una probabilidad del $90 \%$ de éxito y un $10 \%$ de probabilidad que los riesgos se produzcan en el proyecto.

Se optó por crear 16 arquitecturas de redes siguiendo los mismos patrones que en la primera red. Al igual que en la etapa anterior, se busca analizar el performance de la red y la medida del error porcentual (mean) para la elección de la mejor arquitectura y representación de los patrones.

\subsubsection{Construcción de la interfaz de predicción de éxito}

Para poder culminar el caso de estudio se procedió a construir una interfaz visual que acepte valores de los indicadores de los factores de riesgo del proyecto como datos de entrada para predecir la métrica de éxito, que en el modelo es el IC descrito en el apartado 5.3. Se eligió construir una interfaz que se programó en el lenguaje de programación Java, y se empleó el IDE Netbeans 7.0 Beta para su desarrollo. La ejecución de esta interfaz requiere JRE 6 (Java Runtime Environment) 
y un sistema operativo que lo soporte. La interfaz visual es un formulario que tiene como parámetros los diez índices especificados para el empleo del modelo. También tiene tres opciones para cerrar la ventana, limpiar los campos y predecir el resultado según los datos ingresados.

\section{Conclusiones}

Esta investigación realizó una prueba de concepto de la arquitectura genérica que actúa en forma autónoma en los procesos de selección de obras públicas, generando un criterio de decisión autómata en caso de empate.

Para el procedimiento de selección Adjudicación Simplificada, en caso de empate se comprobó la pertinencia de dicha arquitectura en la elección del postor mediante un sorteo electrónico basado en un sistema de aleatoriedad controlada de encriptación y transformación. El método de encriptación usado fue SHA256.

Para el procedimiento de selección Licitación Pública, en caso de empate también se comprobó la idoneidad de la arquitectura en la elección del postor mediante un índice de cumplimiento pronosticado de acuerdo con el comportamiento de la empresa en la ejecución de proyectos de infraestructura similares. En este caso se generó un modelo que realiza la predicción de la probabilidad de cumplimiento (éxito o fracaso) del postor de ejecutar el proyecto. Se estableció un mecanismo para el diseño de indicadores históricos de medición, determinando los factores de riesgo que impactan en los objetivos del proyecto y logrando generar un proceso inteligente y predictivo.

El caso de estudio asociado a Licitación Pública utilizó un conjunto real de observaciones, conformado por la información de los proyectos ejecutados por un grupo empresarial peruano. Se desarrolló una interfaz de predicción de éxito que acepta valores de los indicadores de los factores de riesgo del proyecto como datos de entrada para predecir la métrica de éxito que en el modelo es el IC. Se usaron para tal fin las redes neuronales artificiales como herramienta de análisis predictivo.

\section{Referencias}

Apaza, M. (2003). Balanced Scorecard: Gerencia estratégica y de valor. Lima: Instituto de Investigaciones del Pacífico.

Basili, V., Caldiera, G. y Rombach, H. D. (1994). The Goal Question Metric Approach. College Park: Departamento de Ciencias de la Computación de la Universidad de Maryland. 
Cantone, G., Sarciá, S. , y Basili, V. (2007). A Statistical Neural Network for Risk Management Process. College Park: Departamento de Ciencias de la Computación de la Universidad de Maryland.

Davis, D. (2000). Investigación en administración para la toma de decisiones. México: Thomson.

Del Carpio, J., y Eyzaguirre, R. (2007). Análisis de riesgo en la evaluación de alternativas de inversión utilizando Crystal Ball. Industrial Data, 10(1), 55-58.

Dixon, J.R. (1970). Diseño en ingeniería: inventiva, análisis y toma de decisiones. México Limusa-Wiley .

González Ramírez, M. R. (2001). Sistemas de información para la empresa. Alicante: Publicaciones de la Universidad de Alicante.

Greenwood, W. (1978). Teoría de decisiones y sistemas de información. México: Trillas.

Herrera, F., Herrera-Viedma, E., Verdegay, J.L. (1996). Direct approach processes in group decision making using linguistic OWA operators. Fuzzy Sets and Systems, 79, 175-190.

Huber, G. P. (1984). Toma de decisiones en la gerencia. México: Trillas.

Isasi, P., y Galván, I. (2004). Redes neuronales artificiales: Un enfoque práctico. Madrid: Pearson Education.

Keen, P. G. W. Scoot-Morton, M. S. (1978). Decision support systems: Organizational perspective. Addison Wesley.

Kohonen, T. (1982). Self-organized formation of topologically correct feature maps. Biological cybernetics, 43(1), 59-69.

Lezama, C. (2007). Indicadores de gestión. Recuperado de https://docplayer.es/49436198Indicadores-de-gestion-cruz-lezama-osain.html

Matonis, J. (31 de agosto del 2012). BitZino And The Dawn Of'Provably Fair' Casino Gaming. Forbes. Recuperado de https://www.forbes.com/sites/jonmatonis/2012/08/31/bitzino-and-thedawn-of-provably-fair-casino-gaming/\#597270027593

Menguzzato, M., Renau, J.J. (1995). La dirección estratégica de la empresa: Un enfoque innovador del management. Barcelona: Ariel.

Michie, D., Spiegelhalter, D. J.y Taylor, C.C. (1994). Machinelearning, neural and statistical classification. Londres: Ellis Horwood.

Moody, P.E. (1991). Toma de decisiones gerenciales. Bogotá: Mc Graw Hill Latinoamericana. 
Rumelhart, D. E., Hinton, G.E. y Williams, R. J. (1986). Learning Internal Representations by Error Propagation. En: Rumelhart, D. E., McClelland, J. L. y The PDP Research Group, Parallel Distributed Processing: Explorations in the Microstructure of Cognition (Vol. 1). Boston: MIT Press.

Salgueiro, A. (2001). Indicadores de gestión y cuadro de mando. Madrid: Ediciones Diaz Santos.

Sarcià, S. A., Cantone, G., y Basili, V. R. (2007). A Statistical Neural Network Framework For Risk Management Process. Procedings of ICSOFT, Barcelona, SP, 2007.

Sarle, W. S. (2002). Ai-faq/neural-nets. Recuperado de ftp://ftp.sas.com/pub/neural/FAQ.html

Serra, D. (2004). Métodos cuantitativos para la toma de decisiones. Madrid: Gestión 2000.

Simon, H.A. (1977). The new science of management decision. New Jersey: Prentice-Hall.

Simon, H.A. (1980). El comportamiento administrativo: Estudio de los procesos decisorios en la organización administrativa. Madrid: Aguilar.

Smith, J. C. (1990). A neural network: could it work for you? Financial Executive, 6(3), 26-30.

Zimmermann, H. J. (1991). Fuzzy sets theory and its application. Boston: Kluwer Academic Publishers. 